\title{
Vida útil en masas y productos derivados del maíz: estudio bibliométrico
}

\author{
Shelf life in dough and corn-derived products: bibliometric \\ study
} Luz Eliana Hernández Montoya ${ }^{1}$, Eduardo Javid Corpas Iguarán ${ }^{1 *}$ (D),
Katherin Castro Ríos ${ }^{1}$ (D)

${ }^{1}$ Universidad Católica de Manizales, Instituto de Investigación en Microbiología y Biotecnología Agroindustrial (IMBA), Manizales - Colombia

*Corresponding Author: Eduardo Corpas Iguarán, Universidad Católica de Manizales, Instituto de Investigación en Microbiología y Biotecnología Agroindustrial (IMBA), Carrera 23, No. 60-63, 00000, Manizales, Caldas -

Colombia, e-mail: ecorpas@ucm.edu.co

Cite as: Hernández Montoya, L. E., Corpas Iguarán, E. J., \& Castro Ríos, K. (2020). Shelf life in dough and cornderived products: bibliometric study. Brazilian Journal of Food Technology, 23, e2019023.

https://doi.org/10.1590/1981-6723.02319

\begin{abstract}
Resumen
El análisis bibliométrico permite hacer un análisis retrospectivo del estado del arte de las producciones científicas de un área temática de interés, posibilitando los niveles de progresión de la investigación, enfocada particularmente a las determinaciones de frecuencia o interacciones de los actores. La finalidad del trabajo fue el análisis bibliométrico de la producción científica sobre la vida útil de masas y productos derivados del maíz, para esto se realizó un estudio descriptivo y cuantitativo de la producción científica contenida en la colección de la base de datos de Web of Science, mediante la aplicación de una ecuación de búsqueda en el periodo temporal 2001 al 2017; empleando indicadores bibliométricos de producción, visibilidad e impacto, relación y colaboración (Índice H); posteriormente se organizaron en las herramientas de BibExcel y VOSviewer e interpretaron mediante análisis de frecuencia. Como resultados, un total de 68 registros cumplieron con los criterios de la ecuación de búsqueda planteada. En el 2017 se presentó la mayor frecuencia de publicaciones, 14 documentos; mientras que en el 2009 presentó el mayor número de citaciones, 112 en total. De los autores más representativos y con mayor índice $\mathrm{H}$ se destaca Constantina Tzia, entre las principales instituciones participante las más relevantes fueron, Texas A\&M University, Jiangnan University, National Technical University of Athens, lowa State University y Sonora University y el país con mayor participación fue Estados Unidos. Se logró percibir que la principal revista para la divulgación del conocimiento en el tema fue Journal of Food Science and Technology-Mysore y el área de investigación fue Ciencia y Tecnología de Alimentos (Food Science Technology). Se concluye que a través de los años las publicaciones y las citaciones sobre el tema han incrementado, los principales estudios en vida útil relacionados con masas y productos derivados de maíz se han centrado en la nixtamalización del maíz, calidad e inocuidad de materias primas y productos procesados y en la estabilidad del maíz en almacenamiento.
\end{abstract}

Palabras clave: Bibliometría; Indicadores bibliométricos; BibExcel; VOSviewer; Maíz; Caducidad. 


\begin{abstract}
A Bibliometric study allows a retrospective analysis of the state of the art of the scientific productions of a thematic area of interest, enabling the levels of research progression, particularly focused on the determinations of frequency or actors interactions. The purpose of the present work was the bibliometric analysis of the scientific production on the corn dough shelf life and products derived from the corn. A descriptive and quantitative study of the scientific production contained in the collection of the Web of Science database was carried out, through the application of a search equation in the temporal period 2001 to 2017, using bibliometric indicators of production, visibility and impact, relationship and collaboration ( $\mathrm{H}$ index); subsequently be organized in the tools of BibExcel and VOSviewer and interpreted by frequency analysis. As a result, a total of 68 records met the research equation criteria. The year of 2017 showed the highest frequency of publications, 14 documents; while in the 2009 presented the largest number of citations, 112 in total. The most representative authors and with the highest $\mathrm{H}$ index stands Constantine Tzia, among the main participating institutions the most outstanding were, Texas A\&M University, Jiangnan University, National Technical University of Athens, lowa State University and Sonora University and the countrie with the greatest participation was the United States. It was realized that the principal journal for the dissemination of knowledge was the Journal of Food Science and technology-Mysore and the research area was Food Science and Technology. It is concluded that over the years the publications and citations on the subject have increased, the main shelf life studies related to corn dough and products have focused on the nixtamalization of maize, quality and safety of materials, raw and processed typical corn products and in the stability of corn in storage.
\end{abstract}

Keywords: Bibliometrics; Bibliometrics indicators; BibExcel; VOSviewer; Maize; Stability.

\title{
1 Introducción
}

El maíz (Zea mays L.) es uno de los cereales más cultivados a nivel mundial, siendo Estados Unidos su principal productor (Singh et al., 2011; Gwirtz \& Garcia-Casal, 2014). Es de gran relevancia en la cultura Latinoamérica dado su origen mexicano, en América del Sur su producción para finales del 2017 se estimaba en 162,5 millones de toneladas (Food and Agriculture Organization, 2017). Dado esto y la diversificación de productos que se obtienen a partir de él, su consumo se ha convertido en una tradición que se conserva desde la época pre hispana.

En Colombia, tiene gran importancia debido a sus cualidades nutricionales y sus diversas formas de uso, ya que es la materia prima para la elaboración de productos alimenticios étnicos como arepas, empanadas, tortas y panes. Por tanto, la elaboración y empleo de las masas de maíz en Colombia y el mundo ha trascendido la elaboración artesanal para convertirse en una actividad industrial (Cuevas et al., 2016).

Con referencia a lo anterior, la estimación de la vida útil de las masas de maíz se ha convertido en un desafío para Colombia y el mundo. Mediante investigaciones se ha evidenciado la influencia de condiciones de almacenamiento, de proceso, de distribución y su impacto en parámetros de textura o crecimiento microbiano en las masas de maíz (Chaves-Lopez et al., 2016; Hernández et al., 2019; Hernández Montoya et al., 2019). Los análisis de vida útil se fundamentan en las herramienta de verificación de calidad que garantizan la inocuidad del producto y, por tanto, permiten conocer el periodo o tiempo de deterioro del alimento después de un proceso de trasformación o conservación, buscando que este sea apto para el consumo y que no genere rechazo o riesgo para el consumidor. Expuesto así, el análisis bibliométrico permite evaluar la producción científica, considerando los estudios métricos de la información que produce un investigador o un grupo de investigadores, mediante métodos matemáticos/estadísticos empleados para evaluar la calidad y cantidad de la literatura científica publicada. Permitiendo ilustrar tendencias de investigación mediante análisis de citas, análisis de revistas, autorías, impacto de publicaciones, afiliación o vinculación de instituciones, palabras claves o descriptores, títulos o resúmenes; así como la contribución nacional e internacional en un campo particular. En efecto, estos estudios facilitan no solo conocer más de cerca el tema en particular, sino cualificar internamente el proceso científico (Recio et al., 2017). 
Mediante la implementación de indicadores bibliométricos se permite cuantificar el comportamiento de la producción bibliográfica y la comunicación científica en cualquier campo de la ciencia, a partir de dos importantes funciones: la descriptiva, en la medida que caracteriza el estado de un sistema y la valorativa, que juzga ese estado, según una perspectiva deseable (González et al., 2015). En referencia a lo anterior, existen diversos criterios de clasificación de la información bibliométrica las cuales convergen en tres elementos que son indicadores de productividad, visibilidad o impacto y colaboración o relación.

Indicadores de productividad, se refiere a la frecuencia de publicación de un investigador, grupo de investigadores, instituciones o países (Ospina, 2009), teniendo como objetivo reflejar su inserción en la comunidad científica, evidencia lo más productivo como las temáticas más destacadas de un área de conocimiento y las principales revistas en las cuales se publica (Alonso et al., 2016).

Indicadores de relación o colaboración, se fundamenta en las autorías o en las citaciones conjuntas, son utilizados en la construcción y la visualización de la colaboración científica, así como las redes de co-citación entre los investigadores, instituciones o países mediante técnicas de mapeo (Alonso et al., 2016), uno de los índices más empleados es el de co-autoría, que calcula el número medio de autores por trabajo, siendo también aplicable el índice de co-autoría institucional (Cabezas \& Torres, 2014).

Indicadores de visibilidad e impacto, miden la influencia de los autores sobre los trabajos publicados mediante la cantidad de citas por publicación, la visibilidad se deriva del medio de publicación de un trabajo mientras que el impacto es aquel que se deriva del propio trabajo científico (Cabezas \& Torres, 2014). En relación a esto (Hirsch, 2005, p. 16569) define el índice H como: "Un científico tiene un índice h, si de todos sus trabajos $h$ reciben al menos h citas cada uno, y el resto tiene como máximo h citas". Tiene la ventaja de extender fácilmente para evaluar revistas, instituciones, países o regiones (Departamento Administrativo de Ciencia Tecnología e Innovación, 2016).

Existen herramientas que permiten el análisis y la visualización de las tendencias o relaciones que se producen entre el conocimiento o los autores, a través de un seguimiento de su evolución en el tiempo o el movimiento entre diversas áreas, entre ella tenemos BibExcel y VOSviewer, la primera es una herramienta muy versátil, creada por el departamento de sociología de la Universidad de Umeå en Suecia; basada en extraer campos, analizar las frecuencias de términos y construir las relaciones de los términos para generar las matrices y vectores que representan ese tipo de análisis, y la segunda es una herramienta de uso libre, desarrollada por la Universidad de Leiden en los Países Bajos, la cual permite la construcción y visualización de redes bibliométricas, estas redes pueden incluir revistas, investigadores o publicaciones individuales y ser construidas a partir de co-citación o acoplamiento bibliográfico y relaciones de coautoría (Pichuante, 2016).

Mediante el desarrollo de un análisis bibliométrico, se planteó como objetivo establecer el comportamiento bibliométrico de las publicaciones sobre la vida útil de masa y productos derivados del maíz y los estudios relacionados con el procesamiento, almacenamiento y materias primas, a partir de una búsqueda en la base de datos Web of Science. A partir de esta búsqueda se pretendía identificar la frecuencia y redes de interacción que involucraban las palabras claves, países, instituciones, revistas y autores en torno al tópico mencionado.

\section{Metodología}

\subsection{Tipo de estudio, material de análisis e hipótesis de trabajo}

El análisis bibliométrico se realizó a partir de la colección de la base de datos de Web of Science de Thomas Reuters debido a su cobertura selectiva de publicaciones con prestigio y visibilidad en las diferentes disciplinas (Archambault et al., 2009); con fecha de acceso a la información el día 5 de abril 2018. 
La ecuación de búsqueda de palabras clave empleada fue: "shelf life" and maize OR "shelf life" and "Zea mays" OR "shelf life" and corn flour" OR "shelf life" and "maize flour" OR "shelf life" and "arepas" OR "shelf life" and empanadas OR "shelf life" and "masa corn" OR "shelf life" and "texture dough", en el periodo de tiempo comprendido entre 2001 y 2017 con la intención de analizar la investigaciones más recientes y ampliar el panorama de la información. Para ello, se consideraron específicamente los documentos de tipología "artículos", "revisiones", o "capítulos de libros" con enfoque en el tema.

Es importante resaltar que la ecuación de búsqueda integró dos productos derivados del maíz, arepas y empanadas, considerando que son los de mayor relevancia cultural y económica en Colombia.

La hipótesis de trabajo fue la siguiente:

Existe un comportamiento destacable de la frecuencia e interacciones, en cuanto a los países, palabras claves, instituciones, revistas y autores involucrados en los estudios sobre vida útil en masas y productos derivados del maíz.

\subsection{Indicadores bibliométricos}

Se utilizaron los siguientes indicadores bibliométricos: Indicadores de producción, como el número de publicaciones respecto al tipo de documento, autores, países, organización o institución, áreas de investigación, revista, año y total de citaciones. Indicadores de visibilidad e impacto, para la determinación del índice $\mathrm{H}$ de los autores, las instituciones y los países. Indicadores de relación y colaboración, para la ejecución de mapas temáticos de co-autorías entre autores y países, y la coocurrencia de palabras claves.

\subsection{Recopilación y análisis de datos}

La recopilación de los datos se obtuvo mediante la descarga de los registros obtenidos usando la ecuación de búsqueda previamente descrita, en formato de texto plano de la base de datos de Web of Science. Se emplearon las herramientas de BibExcel y VOSviewer para organizar y clasificar los indicadores bibliométricos, posterior se aplicaron los cálculos de frecuencia y las representaciones mediante tablas y gráficas en el programa de Microsoft Excel® 2013.

\section{Resultados y discusión}

En este estudio se identificaron un total de 115 documentos, aplicando los criterios de la ecuación de búsqueda de palabras claves, se filtraron un total de 68 documentos a la fecha de consulta. A partir de los datos obtenidos se determinó que el artículo con mayor número de citaciones fue: "Sourdough in gluten-free bread-making: An ancient technology to solve a novel issue?", escrito por: Moroni AV; Dal Bello F; Arendt EK, con un total de 100 citaciones, publicado en la revista Food Microbiology en el año 2009 (Moroni et al., 2009).

\subsection{Indicadores de producción}

El artículo original fue la tipología documental de mayor frecuencia representando el 93\% (63 artículos) de los documentos, y el 7\% restante correspondió a revisiones de tema. La Figura 1 muestra la evolución de la productividad científica a partir del año 2001 hasta el 2017; donde se evidencia una tendencia creciente de las publicaciones sobre el tema, a inicios del año 2002 con publicaciones que contemplan las condiciones y características de la tortillas de maíz nixtamalizado, un producto derivado del maíz que es de gran consumo en la cocina mexicana (Book et al., 2002; Seetharaman et al., 2002). Después del año 2014 se evidencia un aumento relativo de las publicaciones (9 publicaciones) hasta el año 2017 (14 publicaciones), este resultado 
puede estar relacionado con el creciente interés mundial de establecer materias primas y alimentos inocuos de la mejor calidad, mediante la constante búsqueda de determinar su vida útil por medio de las condiciones fisicoquímicas y microbiológicas de los productos. En relación al número de citaciones se puede observar un aumento entre el periodo del 2008 al 2012, y se evidencia una ausencia de citación en el año 2010 posiblemente se relacione al único documento de ese año, que hasta la fecha de consulta no contaba con citaciones; entre los cuales sobresalen temas como la estabilidad en el almacenamiento de los granos maíz frente aspecto toxicológicos (Luna-Vital et al., 2017) y la estabilidad de los extractos de los granos de maíz morado cuando se someten a diferentes $\mathrm{pH}$ (Temba et al., 2017).

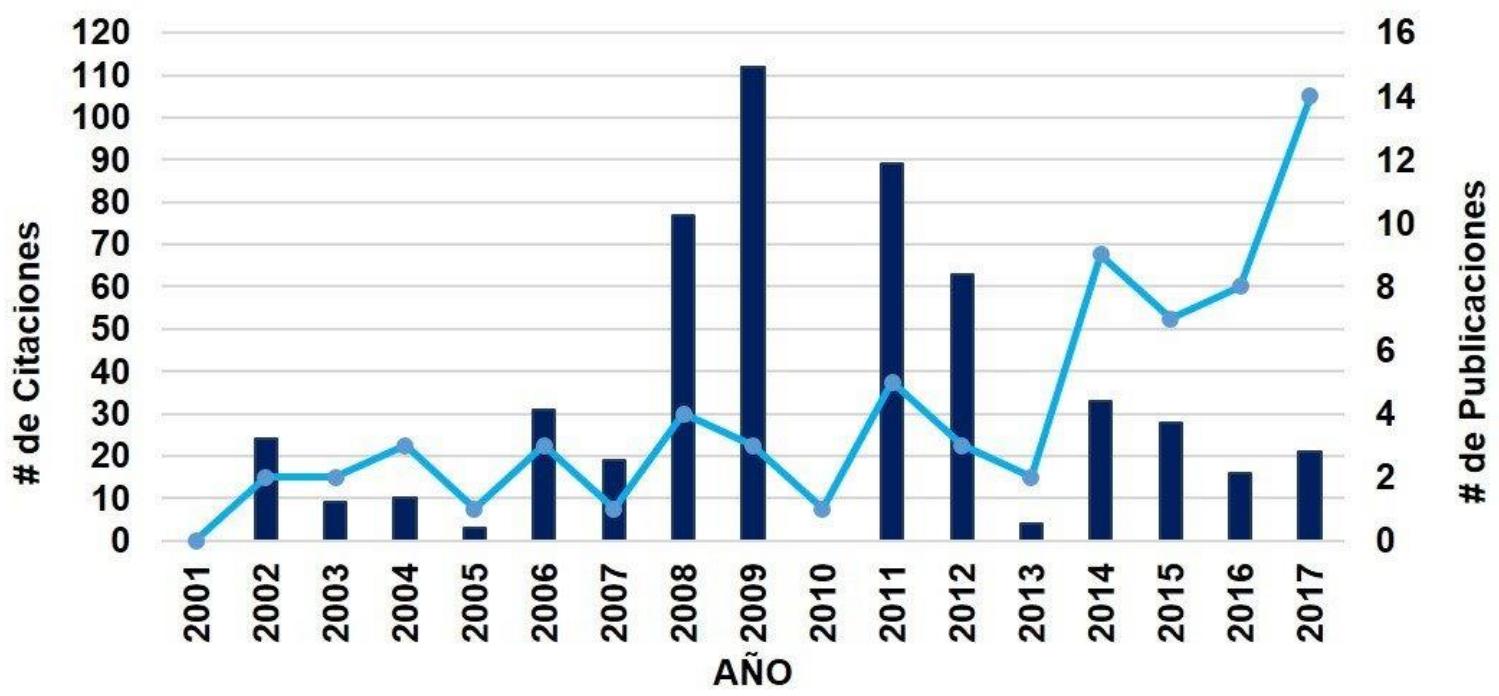

CITACIONES $\rightarrow$ PUBLICACIONES

Figura 1. Número de publicaciones respecto al número de citaciones por año.

\subsubsection{Instituciones}

Durante los procesos de investigación la intervención de las instituciones u organizaciones juegan un papel fundamental en la ejecución y divulgación del conocimiento, en consecuencia la Figura 2 muestra las 15 instituciones con mayor participación en relación al mayor número de publicaciones sobre el tema de búsqueda, fueron: Texas A\&M University (Seetharaman et al., 2002; Mondal et al., 2008; SantanaGálvez et al., 2016), Jiangnan University (Zhou et al., 2016; Sharif et al., 2017; Yue et al., 2017), National Technical University of Athens (Sabanis et al., 2009; Lebesi \& Tzia, 2011; Sabanis \& Tzia, 2011), Iowa State University (Seetharaman et al., 2002; Vidal-Quintanar et al., 2003; Antunez et al., 2012) y Sonora University (Vidal-Quintanar et al., 2003; Márquez-Castillo \& Vidal-Quintanar, 2011; SánchezMadrigal et al., 2015); resaltando las dos primeras instituciones como aquellas que presentaron publicaciones en los últimos dos años, de igual manera sobresale la participación de CSIR, University Minnesota, University of Johannesburg, Brigham Young University y University Auckland con publicaciones en el año 2017. Cuando ocurre una interacción entre una o más instituciones se convierte en un pilar para el intercambio y multidisciplinariedad del conocimiento científico como ocurre entre Texas A\&M University y Iowa State University que generan conocimiento acerca las propiedades de textura, viscosidad y térmicas de dos productos horneados comunes hechos de harina de trigo, panecillos y tortillas de maíz (Seetharaman et al., 2002); y también la interacción que presenta esta última institución con Sonora University en contribuir con la información en temas de vida útil en masa de maíz nixtamalizadas con procesos de autooxidación (VidalQuintanar et al., 2003). 


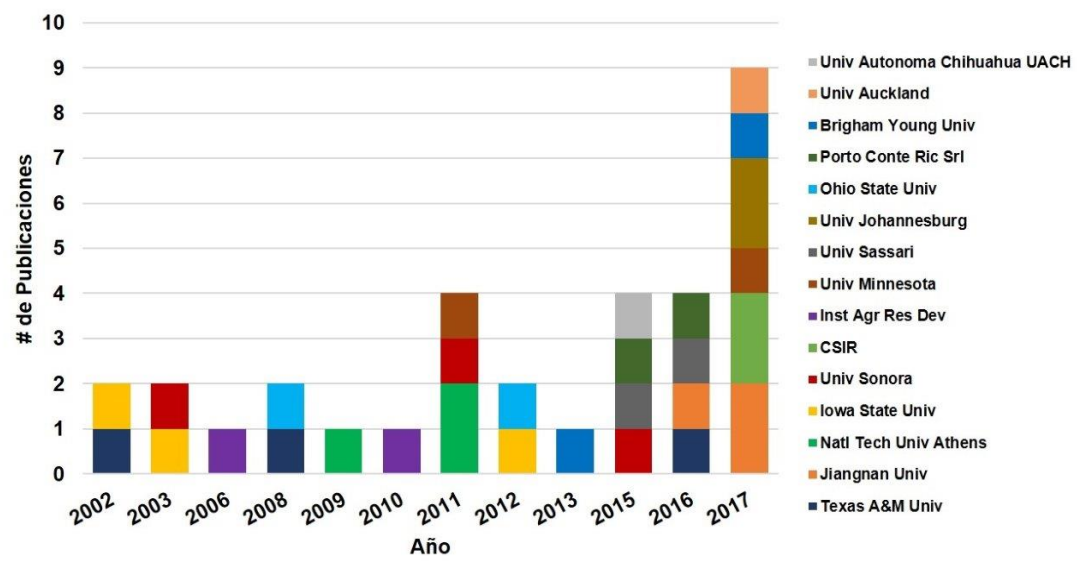

Figura 2. Principales instituciones participantes en relación al número y año de publicación.

\subsubsection{Colaboración internacional}

Determinar el país de origen o las colaboraciones entre ellos para la generación de nuevos conocimientos, soportan el enfoque de la investigación y sus posibles potenciales investigativos, siendo así, se contabilizaron un total de 28 países participantes. En la Figura 3 podemos evidenciar los 10 países con mayor participación, resaltando principalmente la participación de Estados Unidos con un 20,5\% entre el periodo de 2011 al 2017, uno de sus trabajos está centrados en caracterizar e identificar la microbiota de masas de maíz nixtamalizada de seis molinos comerciales diferentes de tortillas en todo Guadalajara, México (Adolphson et al., 2013) y un segundo trabajo enfocado en una revisión de los residuos del procesamiento de masa de maíz: generación, propiedades y utilización potencial con el fin de desarrollar una base de conocimiento sobre los residuos generados (Rosentrater, 2006). Posteriormente sigue la India con 13,2\% entre el periodo de 2013 al 2017; algunas de sus publicaciones se apoyan en la evaluación y monitoreo de la condiciones fisicoquímicas y sensoriales de alimentos típicos de la india cuando son almacenados a diferentes temperaturas de conservación, con el fin de establecer su vida útil a través del tiempo (Singh et al., 2013; Mogra \& Choudhry, 2014; Kaushik et al., 2015); luego China con 11,8\% entre el periodo del 2014 al 2017; México con 10,3\%entre el periodo de 2011 al 2017 e Italia con 8,8\% en los años 2015 y 2016; de allí en menor participación el resto de países. De igual forma, se destaca que entre los países con mayor número de citaciones fue Irlanda y Grecia con 128 y 71 citas respectivamente.

No obstante, se identifica la participación de Colombia trabajando en conjunto con Argentina en el artículo titulado "Antifungal effect of kéfir fermented milk and shelf life improvement of corn arepas" (Gamba et al., 2016). De acuerdo a todo lo anterior podemos evidenciar que durante los primeros once años la participación de los países en general fue relativamente baja y constante, y solo hasta el año 2014 se refleja una tendencia de aumento en la intervención de los países.

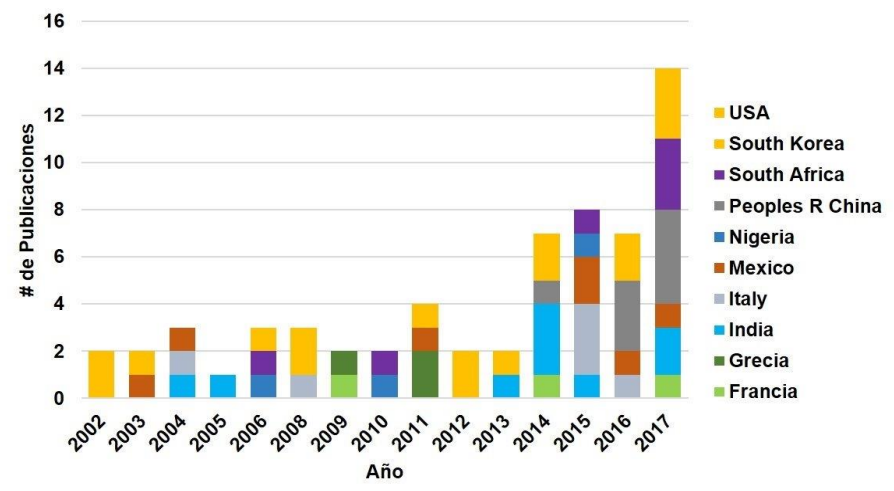

Figura 3. Participación de países con relación al número y año de publicación. 


\subsubsection{Revistas de publicación}

Las revistas pueden actuar como agentes en el proceso científico en forma de canales los cuales pueden poner en consenso la información en común del conocimiento científico; de acuerdo a esto se encontraron un total de 44 revistas, en la Figura 4 se pueden identificar las revistas con mayor número de referencias o publicaciones, los cuales coinciden en temas relacionados con alimentos, cereales, microbiología, y química de alimentos resaltando las siguientes: Journal of Food Science and Technology-Mysore con el 10,3\%, una de sus publicaciones comenta sobre la influencia del almacenamiento de la harina de maíz en la calidad proteica, funcional y sensorial (Shobha et al., 2014), en un análisis bibliométrico aplicado a esta revista se observó un aumento en el número de contribuciones en volúmenes sucesivos, siendo India el principal contribuyente tanto en el volumen inaugural como en los cinco volúmenes estudiados y el mayor número de contribuciones es de los autores conjuntos (Vijay \& Raghavan, 2007). Continuando con Cereal Chemistry con 5,9\% y Journal of Food Processing and Preservation con 5,9\%, además de European Food Research and Technology con 5,9\% y por ultimo Journal of Cereal Science con 4,4\%.

Así mismo, se determinó una tendencia creciente a partir del año 2014 del número de publicaciones realizadas en las revistas de mayor impacto para esta área de conocimiento, exponiendo que entre los temas publicados en el último año estaban basados en aspectos de la estabilidad de la vida útil y la calidad de productos a base de maíz como masas y tortillas mediante la adición de conservantes proteicos (Phillips et al., 2017; Yue et al., 2017) y en las condiciones reológicas y microbiológicas del grano y las masas de maíz que favorecen la vida anaquel (Deepa \& Umesh Hebbar, 2017b; Valderrama-Bravo et al., 2017).

A partir de las revistas se logró sondear y obtener las principales áreas de investigación de mayor influencia, resaltando principalmente el área: Food Science Technology con 53 documentos (57\%), posteriormente las otras áreas como, Chemistry Applied con 8 documentos (9\%) y Biotechnology Applied Microbiology con 6 documentos (7\%), de modo que con el trascurso de los años se observa una tendencia de aumento constante del número publicaciones en esta principal área de conocimiento, de manera que enfoca las investigaciones en alimentos desde el punto de vista de sus propiedades y composiciones (físicas, químicas y microbiológicas) hasta su desarrollo o mejoramiento a nivel industrial.

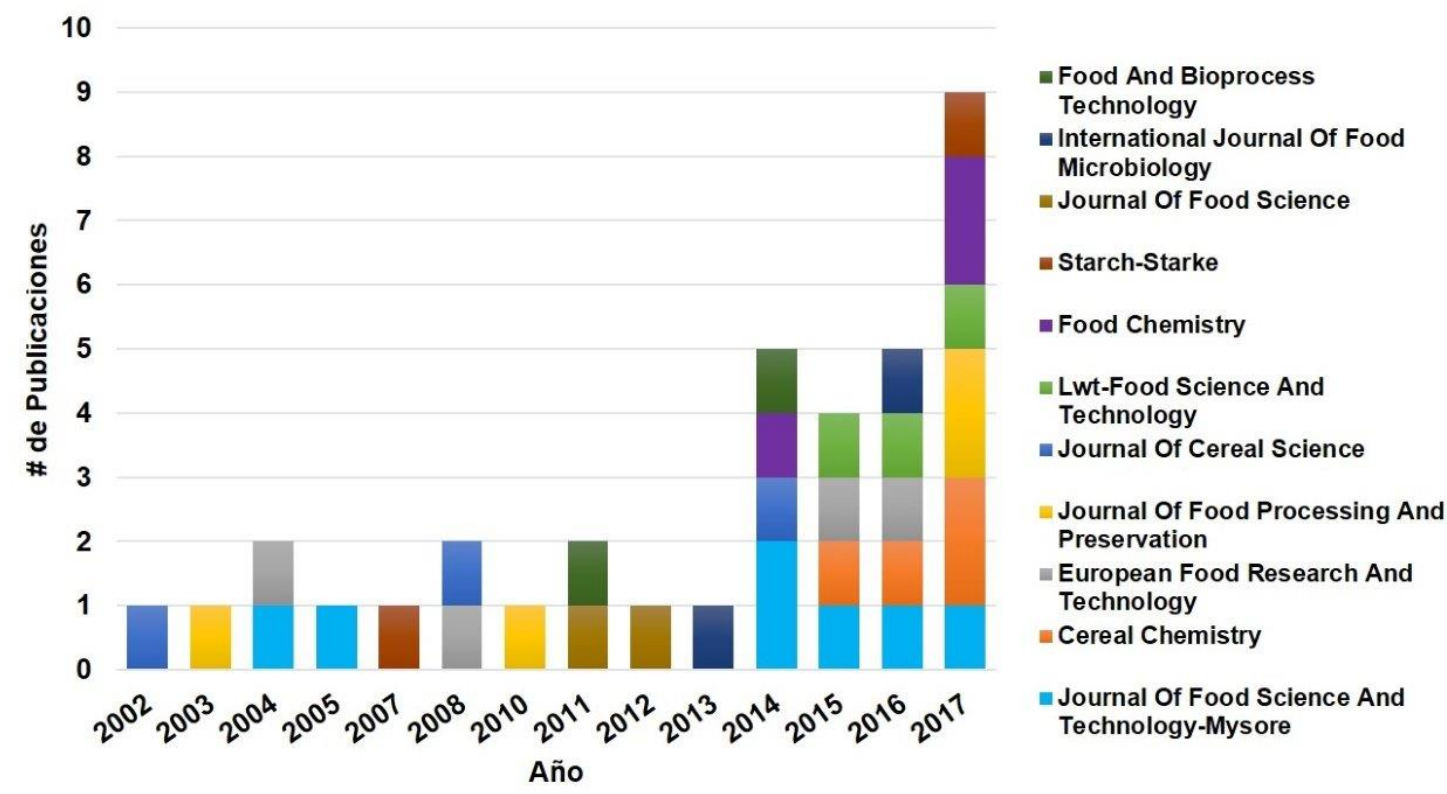

Figura 4. Principales revistas en relación al número y año de publicación. 


\subsection{Indicadores de visibilidad}

Los 68 documentos analizados fueron publicados por un total de 274 autores. Uno de los autores con mayor relevancia en las investigaciones sobre el tema con respecto a los documentos encontrados; es la profesora Constantina Tzia del departamento de Ingeniería Química de la Universidad Nacional de Atenas en Grecia, con un total de 3 publicaciones, un índice $\mathrm{H}$ de 3 y 71 citaciones. La Tabla 1 muestra los autores y su correspondiente índice $\mathrm{H}$, marcando la productividad y evaluando el impacto en el acumulado y relevancia de las publicaciones, analizando la correlación entre la producción científica generada y la cantidad de citas recibidas de su producción académica en relación al tema, aunque se registran índices relativamente bajos, esto se asocia a la baja producción científica relacionada con el tema durante el periodo de estudio; no obstante se resalta el trabajo en conjunto de los autores Deepa, C y Hebbar, HU cuyos temas se enfocan en los procesos de micronización (jet-milling) sobre los granos de maíz con posterior transformación a harina de maíz para conservar su vida útil (Deepa \& Umesh Hebbar, 2014; Deepa \& Umesh Hebbar, 2017a, 2017b).

De los autores más citados encontramos a Arendt EK con 2 publicaciones y un total de 128 citaciones (Moroni et al., 2009; Zannini et al., 2012) y de la contribución de autores por documento se encontró que

de todos los documentos solo uno contaba con la participación de 9 autores (Sharif et al., 2017) y 3 documentos con la participación de 8 autores cada una (Okeke et al., 2015; Sánchez-Madrigal et al., 2015; Valderrama-Bravo et al., 2017).

También se determinó el índice $\mathrm{H}$ de las instituciones y los países participantes donde se evidencia que las instituciones National Technical University of Athens y Iowa State University presentaron mayor indice $H: 3$ y Estados unidos el país con mayor índice H:6.

Tabla 1. Principales autores y su índice $H$.

\begin{tabular}{cccccc}
\hline Autor & Publicaciones & País & H- index & Citaciones & Referencia \\
\hline Tzia C & 3 & Grecia & 3 & 71 & $\begin{array}{c}\text { Sabanis et al. (2009), Lebesi } \\
\text { \& Tzia (2011), Sabanis \& } \\
\text { Tzia (2011) }\end{array}$ \\
\hline Deepa C & 3 & India & 1 & 4 & $\begin{array}{c}\text { Deepa \& Umesh Hebbar } \\
\text { (2014, 2017a, 2017b) }\end{array}$ \\
\hline Hebbar HU & 3 & India & 1 & 4 & $\begin{array}{c}\text { Deepa \& Umesh Hebbar } \\
\text { (2014, 2017a, 2017b) }\end{array}$ \\
\hline Perez-Carrillo E & 2 & México, USA & 1 & 3 & $\begin{array}{c}\text { Chuck Hernández et al. } \\
\text { (2015), Santana- } \\
\text { Gálvez et al. (2016) }\end{array}$ \\
\hline Piga A & 2 & Italia & 2 & 6 & $\begin{array}{c}\text { Sanguinetti et al. (2015, } \\
\text { 2016) }\end{array}$ \\
\hline Del Caro A & 2 & Italia & 2 & 6 & $\begin{array}{c}\text { Sanguinetti et al. (2015), } \\
\text { Sanguinetti et al. (2016) }\end{array}$ \\
\hline Waniska RD & 2 & USA & 2 & 31 & $\begin{array}{c}\text { Seetharaman et al. (2002), } \\
\text { Mondal et al. (2008) }\end{array}$ \\
\hline Catzeddu P & 2 & 2 & 6 & $\begin{array}{c}\text { Sanguinetti et al. (2015), } \\
\text { Sanguinetti et al. (2016) }\end{array}$ \\
\hline
\end{tabular}

\subsection{Indicadores de relación}

Mediante el empleo de la herramienta VOSwiever se realizó la construcción del mapeo de redes sobre coautorías entre autores indicando que de 274 autores solo 11 de ellos trabajaron en co-autorías. En la Figura 5 
se muestran cinco clústeres donde 3 de ellos trabajan temas en conjunto; la relación más estrecha se presenta en el primer clúster que concierne a cinco autores entre ellos con publicaciones en los años 2015-2016, seguido del segundo y tercer clúster que muestra la correlación entre dos autores y publicaciones en el año 2015 y 2010 , respectivamente.

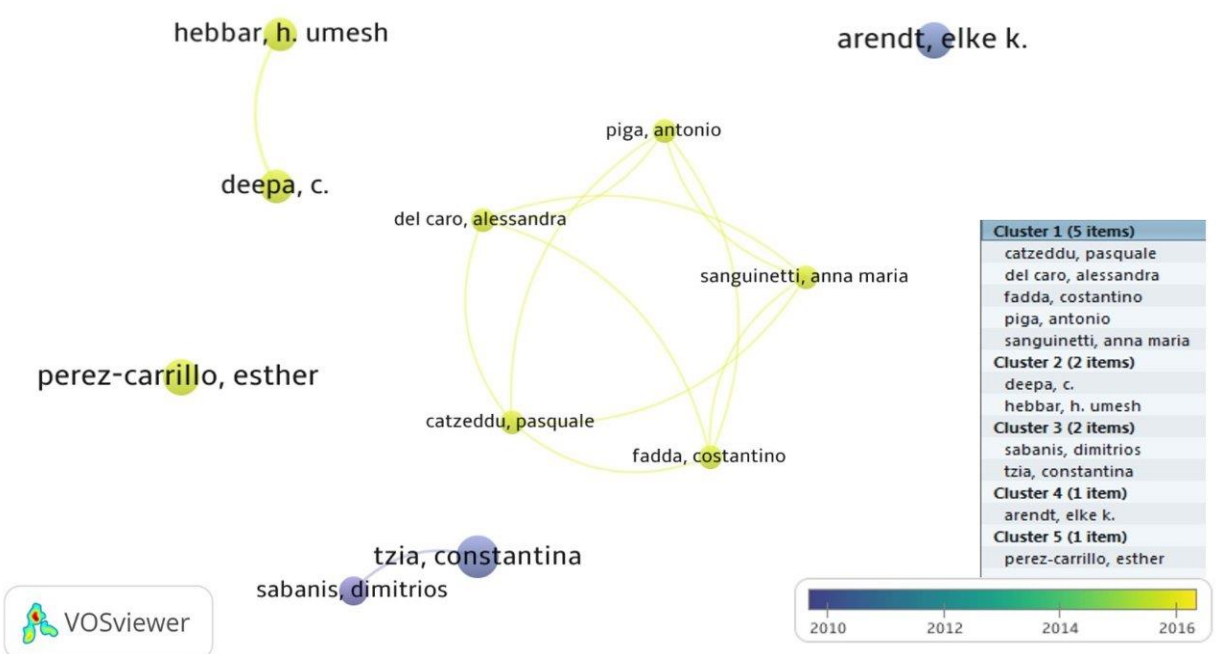

Figura 5. Mapa de redes de co-autorías entre autores.

No obstante en la Figura 6, se detalla la interpretación de la relación de co-autorías entre países, deduciendo que de los 28 países, tan solo 7 de ellos han trabajo bajo co-autoría de las publicaciones, siendo Estado Unidos el más representativo y trabajando en conjunto con países como Francia (Hesso et al., 2014; Liu et al., 2017), Italia (Clubbs et al., 2008), México (Vidal-Quintanar et al., 2003; Santana-Gálvez et al., 2016) y China (Zheng et al., 2016), como se refleja en el primer clúster; otros países que trabajan en relación de co-autorías son Sudáfrica y Nigeria (Ugoji et al., 2006; Darman Djoulde et al., 2010; Okeke et al., 2015); el resto de países lo hacen de carácter independiente.

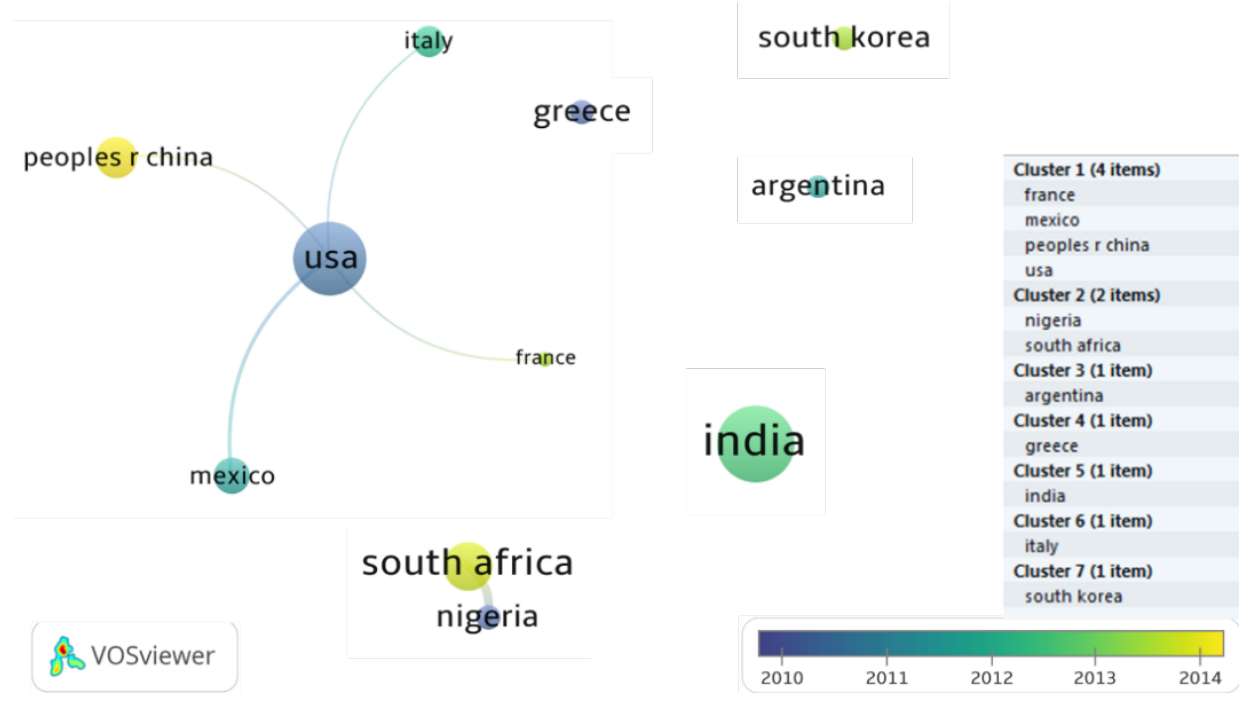

Figura 6. Mapa de redes de co-autorías entre países. 
A partir del análisis de co-ocurrencia de las palabras claves en la Figura 7 se puede inferir que del total de 554 palabras claves, 17 de ella se encontraban concurrentemente y presentaban una relación estrecha sobre el tema. Para ello, se obtuvo un total de 17 nodos agrupados en 3 clúster que conectan entre sí con 147 enlaces, determinando lo siguiente:

- Los nodos rojos reflejan las relaciones más dinámicas, donde la principal palabra empleada en el mayor porcentaje de documentos encontrados es "Maize" en afinidad con "Shelf life", "Shelf-life", "Quality" y "Storage"; teniendo en cuenta que en algunos documentos la escritura de la palabra "Shelf life" puede variar por un guion;

- En los nodos azules se evidencia una relación muy fuerte entre las palabras claves "Dough" y "Texture", dado que en los estudios de las condiciones reológicas y textura de las masas estas propiedades son factores asociados que definen la influencia de los diferentes componentes, permitiendo el control de calidad del producto y orientando al diseño y adaptación de nuevas tecnologías;

- Por otro lado, los nodos verdes tratan palabras que hacen referencia a factores que tiene influencia en los estudios de vida útil de los alimentos como lo son "Temperature".

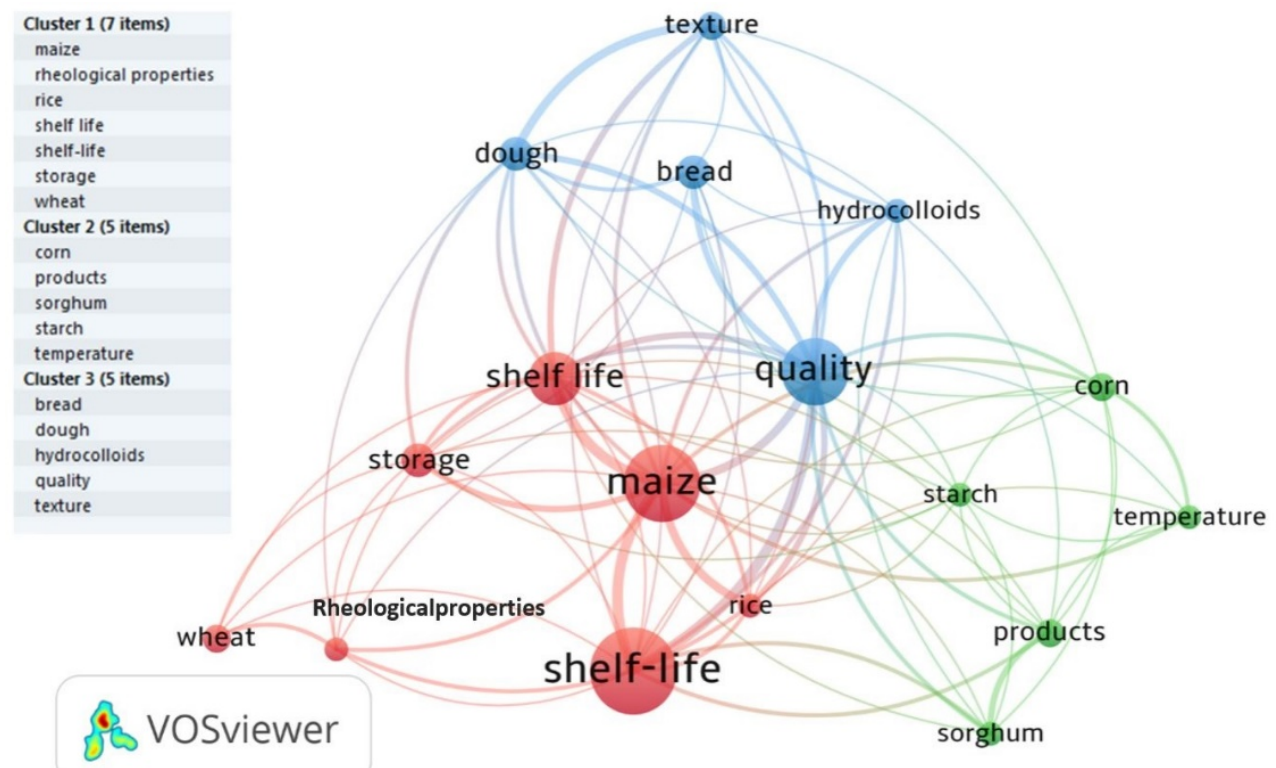

Figura 7. Mapeo de co-ocurrencia de palabras claves.

\section{Conclusiones}

Se evidenció un crecimiento gradual en los últimos años de la información científica; los principales estudios sobre vida útil relacionados con masas y productos derivados de maíz se han centrado en la nixtamalización del maíz, calidad e inocuidad de materias primas y productos procesados y en la estabilidad del maíz en almacenamiento. Se identificó una gran participación de países de diversos continentes como Estados Unidos, Italia, China, India y Sudáfrica; al igual que de instituciones reconocidas como Texas A\&M University, Jiangnan University, National Technical University of Athens, Iowa State University y Sonora University, partiendo de la importancia que se genera a partir de las relaciones entre co-autorías cuando se ejecutan trabajos e investigaciones en conjunto con otros países e instituciones para la generación del nuevo conocimiento fundamentado en las propiedades y composición del maíz, junto con el apoyo de importantes revistas que sirven como canal para transmitir la información como Journal of Food Science and TechnologyMysore. 
Además, se justificó la participación conjunta entre actores investigando sobre el tema desde diferentes aspectos, generando un impacto por parte de autores como Constantina Tzia la de mayor índice $\mathrm{H}$ y Arendt EK como el de mayor citación. Finalmente, es importante destacar a los actores e identificar lo que han trabajado puede generar perspectivas para el desarrollo de futuras investigaciones enfocadas en el mejoramiento de la calidad, e inocuidad del maíz y sus derivados y a su vez involucrar diferentes procesos, técnicas o metodologías para la ejecución de los mismos.

\section{Agradecimientos}

Los autores expresan agradecimientos a Colciencias por la financiación y la Universidad Católica de Manizales por la co-financiación de la joven investigadora Luz Eliana Hernandez Montoya mediante la convocatoria de jóvenes investigadores e innovadores por la paz 2017.

\section{Referencias}

Adolphson, S. J., Dunn, M. L., Jefferies, L. K., \& Steele, F. M. (2013). Isolation and characterization of the microflora of nixtamalized corn masa. International Journal of Food Microbiology, 165(3), 209-213. PMid:23803568. http://dx.doi.org/10.1016/j.ijfoodmicro.2013.05.010

Alonso, A., Tannuri de Oliveira, E. F., Cabrini, M. C., Pandiella, A., \& Benavent, R. A. (2016). Un análisis bibliométrico en el área de la Medicina: colaboración científica entre Brasil y España (2002-2011). Investigación Bibliotecológica: Archivonomía, Bibliotecología e Información, 30, 205-230. http://dx.doi.org/10.1016/J.IBBAI.2016.04.018

Antunez, P. D., Botero Omary, M., Rosentrater, K. A., Pascall, M., \& Winstone, L. (2012). Effect of an oxygen scavenger on the stability of preservative-free flour tortillas. Journal of Food Science, 77(1), S1-S9. PMid:22133586.

http://dx.doi.org/10.1111/j.1750-3841.2011.02470.x

Archambault, É., Campbell, D., Gingras, Y., \& Larivière, V. (2009). Comparing bibliometric statistics obtained from the Web of Science and Scopus. Journal of the American Society for Information Science and Technology, 60(7), 1320-1326. http://dx.doi.org/10.1002/asi.21062

Book, S. L., Brill, R. V., \& Heidolph, B. B. (2002). Effects of leavening acids on characteristics of fresh and 30-day-old tortillas. Cereal Foods World, 47, 390.

Cabezas, A., \& Torres, D. (2014). Manual introductorio: Políticas científicas e indicadores bibliométricos. Granada, España.

Chaves-Lopez, C., Serio, A., Delgado-Ospina, J., Rossi, C., Grande-Tovar, C. D., \& Paparella, A. (2016). Exploring the bacterial microbiota of colombian fermented maize dough "Masa Agria" (Maiz añejo). Frontiers in Microbiology, 7, 1168. PMid:27524979. http://dx.doi.org/10.3389/fmicb.2016.01168

Chuck Hernández, C., Perez Carrillo, E., Soria Hernández, C., \& Serna Saldívar, S. O. (2015). Functionality and organoleptic properties of maize tortillas enriched with five different soybean proteins. Cereal Chemistry Journal, 92(4), 341-349. http://dx.doi.org/10.1094/CCHEM-07-14-0154-R

Clubbs, E. A., Vittadini, E., Shellhammer, T. H., \& Vodovotz, Y. (2008). Effects of storage on the physico-chemical properties of corn tortillas prepared with glycerol and salt. Journal of Cereal Science, 47(2), 162-171. http://dx.doi.org/10.1016/j.jcs.2007.03.007

Cuevas, J. F., Plaza, J. L., \& Fernández, A. (2016). Análisis de perfil de textura en masas reconstituidas de harina de maíz amarillo fermentado. Agronomia Colombiana, 34, 537-539. http://dx.doi.org/10.15446/agron.colomb.v34n1supl.58339

Darman Djoulde, R., Oldewage-Theron, W., Egal, A. A., \& Samuel, F. (2010). Influence of room-temperature storage-reheating cycles on nutritional properties of maize meal porridge (pap) used in the VAAL region, South Africa. Journal of Food Processing and Preservation, 34(2), 181-191. http://dx.doi.org/10.1111/j.1745-4549.2008.00323.x

Deepa, C., \& Umesh Hebbar, H. (2014). Micronization of maize flour: Process optimization and product quality. Journal of Cereal Science, 60(3), 569-575. http://dx.doi.org/10.1016/j.jcs.2014.08.002

Deepa, C., \& Umesh Hebbar, H. (2017a). Influence of micronization on physicochemical properties of maize grains. Stärke, 69(3-4), 1600060. http://dx.doi.org/10.1002/star.201600060

Deepa, C., \& Umesh Hebbar, H. (2017b). Effect of micronization of maize grains on shelf-life of flour. Journal of Food Processing and Preservation, 41(5), e13195. http://dx.doi.org/10.1111/jfpp.13195

Departamento Administrativo de Ciencia Tecnología e Innovación - COLCIENCIAS. (2016). Política para mejorar la calidad de las publicaciones científicas nacionales (92 p.). Bogotá D.C

Food and Agriculture Organization - FAO. (2017). Producción de cereales de América Latina y el Caribe en 2017 superó en 20 por ciento el nivel de 2016. Rome.

Gamba, R. R., Caro, C. A., Martínez, O. L., Moretti, A. F., Giannuzzi, L., De Antoni, G. L., \& León Peláez, A. (2016). Antifungal effect of kefir fermented milk and shelf life improvement of corn arepas. International Journal of Food Microbiology, 235, 85-92. PMid:27447094. http://dx.doi.org/10.1016/j.ijfoodmicro.2016.06.038 
González, M. J. P., Guzmán, M. F., \& Chaviano, O. G. (2015). Criterios, clasificaciones y tendencias de los indicadores bibliométricos en la evaluación de la ciencia. Revista Cubana de Información en Ciencias de la Salud, 26, 290-309. http://dx.doi.org/10.1021/jp907259e

Gwirtz, J. A., \& Garcia-Casal, M. N. (2014). Processing maize flour and corn meal food products. Annals of the New York Academy of Sciences, 1312(1), 66-75. PMid:24329576. http://dx.doi.org/10.1111/nyas.12299

Hernández Montoya, L. E., Agudelo Buritica, M. F., Corpas Iguarán, E., \& Castro-Ríos, K. (2019). Evaluación de conservación y procesamiento en la calidad fisicoquímica y microbiológica de empanadas de maíz. Alimentos Hoy, 27, 3-14.

Hernández, L. E., Peláez, A. C., \& Castro-Ríos, K. (2019). Influence of refrigeration and freezing storage on the instrumental texture of corn dough and empanadas. Contemporary Engineering Sciences, 12(4), 149-156. http://dx.doi.org/10.12988/ces.2019.9620

Hesso, N., Loisel, C., Chevallier, S., \& Le-Bail, A. (2014). Impact of pregelatinized starches on the texture and staling of conventional and degassed pound cake. Food and Bioprocess Technology, 7(10), 2923-2930. http://dx.doi.org/10.1007/s11947014-1254-5

Hirsch, J. E. (2005). An index to quantify an individual's scientific research output. Proceedings of the National Academy of Sciences of the United States of America, 102(46), 16569-16572. PMid:16275915. http://dx.doi.org/10.1073/pnas.0507655102

Kaushik, P., Mishra, A., Malik, A., \& Sharma, S. (2015). Production and shelf life evaluation of storable myco-granules for multiple environmental applications. International Biodeterioration \& Biodegradation, 100, 70-78.

http://dx.doi.org/10.1016/j.ibiod.2015.02.011

Lebesi, D. M., \& Tzia, C. (2011). Effect of the addition of different dietary fiber and edible cereal bran sources on the baking and sensory characteristics of cupcakes. Food and Bioprocess Technology, 4(5), 710-722. http://dx.doi.org/10.1007/s11947-0090181-3

Liu, T., Hou, G. G., Cardin, M., Marquart, L., \& Dubat, A. (2017). Quality attributes of whole-wheat flour tortillas with sprouted whole-wheat flour substitution. Lebensmittel-Wissenschaft + Technologie, 77, 1-7. http://dx.doi.org/10.1016/j.Iwt.2016.11.017

Luna-Vital, D., Li, Q., West, L., West, M., \& Gonzalez De Mejia, E. (2017). Anthocyanin condensed forms do not affect color or chemical stability of purple corn pericarp extracts stored under different pHs. Food Chemistry, 232, 639-647. PMid:28490122. http://dx.doi.org/10.1016/j.foodchem.2017.03.169

Márquez-Castillo, A., \& Vidal-Quintanar, R. L. (2011). Improvements in the Shelf Life of Commercial Corn Dry Masa Flour (CMF) by reducing lipid oxidation. Journal of Food Science, 76(2), C236-C241. PMid:21535741. http://dx.doi.org/10.1111/j.1750-3841.2010.01983.x

Mogra, R., \& Choudhry, M. (2014). Development and quality evaluation of value added instant rab mixes. Journal of Food Science and Technology, 51(6), 1140-1146. PMid:24876647. http://dx.doi.org/10.1007/s13197-012-0615-1

Mondal, S., Tilley, M., Alviola, J. N., Waniska, R. D., Bean, S. R., Glover, K. D., \& Hays, D. B. (2008). Use of near-isogenic wheat lines to determine the glutenin composition and functionality requirements for flour tortillas. Journal of Agricultural and Food Chemistry, 56(1), 179-184. PMid:18072743. http://dx.doi.org/10.1021/jf071831s

Moroni, A. V., Dal Bello, F., \& Arendt, E. K. (2009). Sourdough in gluten-free bread-making: an ancient technology to solve a novel issue? Food Microbiology, 26(7), 676-684. PMid:19747600. http://dx.doi.org/10.1016/j.fm.2009.07.001

Okeke, C. A., Ezekiel, C. N., Nwangburuka, C. C., Sulyok, M., Ezeamagu, C. O., Adeleke, R. A., Dike, S. K., \& Krska, R. (2015). Bacterial diversity and mycotoxin reduction during maize fermentation (steeping) for ogi production. Frontiers in Microbiology, 6 , 1402. PMid:26697001. http://dx.doi.org/10.3389/fmicb.2015.01402

Ospina, D. N. (2009). Caracterización de la producción cinetífica y visibilidad de los investigadores de Universidad Nacional de Colombia sede Medellín en la ISI Web of Science (1990-2007) (Tesis). Universidad Nacional de Colombia, Medellín.

Oteiza, J. M., Giannuzzi, L., \& Lupano, C. E. (2003). Modeling of microbial growth in refrigerated doughs for "empanadas" with potassium sorbate and whey protein concentrate. Journal of Food Processing and Preservation, 27(4), 253-270. http://dx.doi.org/10.1111/j.1745-4549.2003.tb00516.x

Phillips, R., Pike, O. A., Eggett, D. L., \& Dunn, M. L. (2017). Folate stability in folic acid enriched corn masa flour, tortillas, and tortilla chips over the expected shelf life. Cereal Chemistry Journal, 94, CCHEM-02-17-003. http://dx.doi.org/10.1094/CCHEM02-17-0037-R

Pichuante, C. (2016). Visualización de grafos de co-autoría y de conocimiento basado en publicaciones científicas, implentada en VOSviewer (Tesis). Pontificia Universidad Católica de Chile, Santiago.

Recio, M. A., Yepes, N., \& Moreno, F. (2017). Análisis bibliométrico de las publicaciones sobre síndrome metabólico en dos revistas biomédicas colombianas de alto impacto. Salutem Scientia Spiritu, 3, 12-21.

Rosentrater, K. A. (2006). A review of corn masa processing residues: Generation, properties, and potential utilization. Waste Management (New York, N.Y.), 26(3), 284-292. PMid:15936936. http://dx.doi.org/10.1016/j.wasman.2005.03.010

Sabanis, D., \& Tzia, C. (2011). Selected structural characteristics of hpmc-containing gluten free bread: A response surface methodology study for optimizing quality. International Journal of Food Properties, 14(2), 417-431. http://dx.doi.org/10.1080/10942910903221604

Sabanis, D., Lebesi, D., \& Tzia, C. (2009). Development of fibre-enriched gluten-free bread: A response surface methodology study. International Journal of Food Sciences and Nutrition, 60(Supl. 4), 174-190. PMid:19330631.

http://dx.doi.org/10.1080/09637480902721196 
Sánchez-Madrigal, M. Á., Neder-Suárez, D., Quintero-Ramos, A., Ruiz-Gutiérrez, M. G., Meléndez-Pizarro, C. O., PiñónCastillo, H. A., Galicia-García, T., \& Ramírez-Wong, B. (2015). Physicochemical properties of frozen tortillas from nixtamalized maize flours enriched with $\beta$-glucans. Food Science and Technology, 35(3), 552-560. http://dx.doi.org/10.1590/1678-457X.6715

Sanguinetti, A. M., Del Caro, A., Scanu, A., Fadda, C., Milella, G., Catzeddu, P., \& Piga, A. (2016). Extending the shelf life of gluten-free fresh filled pasta by modified atmosphere packaging. Lebensmittel-Wissenschaft + Technologie, 71, 96-101. http://dx.doi.org/10.1016/j.Iwt.2016.03.010

Sanguinetti, A. M., Secchi, N., Del Caro, A., Fadda, C., Fenu, P. A. M., Catzeddu, P., \& Piga, A. (2015). Gluten-free fresh filled pasta: The effects of xanthan and guar gum on changes in quality parameters after pasteurisation and during storage. Lebensmittel-Wissenschaft + Technologie, 64(2), 678-684. http://dx.doi.org/10.1016/j.lwt.2015.06.046

Santana-Gálvez, J., Pérez-Carrillo, E., Velázquez-Reyes, H. H., Cisneros-Zevallos, L., \& Jacobo-Velázquez, D. A. (2016). Application of wounding stress to produce a nutraceutical-rich carrot powder ingredient and its incorporation to nixtamalized corn flour tortillas. Journal of Functional Foods, 27, 655-666. http://dx.doi.org/10.1016/j.jff.2016.10.020

Seetharaman, K., Chinnapha, N., Waniska, D., \& White, P. (2002). Changes in textural, pasting and thermal properties of wheat buns and tortillas during storage. Journal of Cereal Science, 35(2), 215-223. http://dx.doi.org/10.1006/jcrs.2001.0428

Sharif, H. R., Abbas, S., Majeed, H., Safdar, W., Shamoon, M., Khan, M. A., Shoaib, M., Raza, H., \& Haider, J. (2017). Formulation, characterization and antimicrobial properties of black cumin essential oil nanoemulsions stabilized by OSA starch. Journal of Food Science and Technology, 54(10), 3358-3365. PMid:28974821. http://dx.doi.org/10.1007/s13197-017-2800-8

Shobha, D., Kumar, H. V. D., Sreeramasetty, T. A., Puttaramanaik, Gowda, K. T., \& Shivakumar, G. B. (2014). Storage influence on the functional, sensory and keeping quality of quality protein maize flour. Journal of Food Science and Technology, 51(11), 3154-3162. PMid:26396307. http://dx.doi.org/10.1007/s13197-012-0788-7

Singh, B. P., Jha, A., Sharma, N., \& Rasane, P. (2013). Optimization of a process and development of a shelf life prediction model for instant multigrain dalia mix. Journal of Food Process Engineering, 36(6), 811-823. http://dx.doi.org/10.1111/jfpe.12050

Singh, N., Singh, S., \& Shevkani, K. (2011). Maize: Composition, bioactive constituents, and unleavened bread. In V. R. Preedy, R. R. Watson \& V. B. Patel (Eds.), Flour and breads and their fortification in health and disease prevention (pp. 89-99). Amritsar, India: Elsevier. http://dx.doi.org/10.1016/B978-0-12-380886-8.10009-1.

Temba, M. C., Njobeh, P. B., \& Kayitesi, E. (2017). Storage stability of maize-groundnut composite flours and an assessment of aflatoxin B1 and ochratoxin A contamination in flours and porridges. Food Control, 71, 178-186. http://dx.doi.org/10.1016/j.foodcont.2016.06.033

Ugoji, E. O., Laing, M. D., \& Hunter, C. H. (2006). An investigation of the shelf-life (storage) of Bacillus isolates on seeds. South African Journal of Botany, 72(1), 28-33. http://dx.doi.org/10.1016/j.sajb.2005.04.001

Valderrama-Bravo, C., Domínguez-Pacheco, F., Hernández-Aguilar, C., Flores-Saldaña, N., Villagran-Ortíz, P., Pérez-Reyes, C., Sánchez-Hernández, G., \& Oaxaca-Luna, A. (2017). Effect of nixtamalized maize with lime water (Nejayote) on rheological and microbiological properties of masa. Journal of Food Processing and Preservation, 41(1), e12748. http://dx.doi.org/10.1111/jpp.12748

Vidal-Quintanar, R.-L., Love, M. H., Love, J. A., White, P. J., \& Johnson, L. A. (2003). Lipid-autoxidation-limited shelf-life of nixtamalized instant corn masa. Journal of Food Lipids, 10(2), 153-163. http://dx.doi.org/10.1111/j.1745-4522.2003.tb00012.x

Vijay, K. R., \& Raghavan, I. (2007). Journal of Food Science and Technology: A bibliometric study. Annals of Library \& Information Studies, 54, 6 .

Yue, F.-L., Guo, X.-N., \& Zhu, K.-X. (2017). Impact of characteristics of different wheat flours on the quality of frozen cooked noodles. Cereal Chemistry Journal, 94(5), 881-886. http://dx.doi.org/10.1094/CCHEM-04-17-0082-R

Zannini, E., Pontonio, E., Waters, D. M., \& Arendt, E. K. (2012). Applications of microbial fermentations for production of glutenfree products and perspectives. Applied Microbiology and Biotechnology, 93(2), 473-485. PMid:22094979. http://dx.doi.org/10.1007/s00253-011-3707-3

Zheng, A., Zhang, B., Zhou, L., \& Wang, S. (2016). Application of radio frequency pasteurization to corn (Zea mays L.): Heating uniformity improvement and quality stability evaluation. Journal of Stored Products Research, 68, 63-72. http://dx.doi.org/10.1016/j.jspr.2016.04.007

Zhou, C.-F., Qian, P., Meng, J., Gao, S.-M., \& Lu, R.-R. (2016). Effect of glycerol and sorbitol on the properties of dough and white bread. Cereal Chemistry Journal, 93(2), 196-200. http://dx.doi.org/10.1094/CCHEM-04-15-0087-R

Financiamiento: Departamento Administrativo de Ciencia, Tecnología e Innovación. Jóvenes investigadores por la paz 